Clinical study

\title{
Neuroangiography patterns of the middle cerebral artery: Study of 554 cerebral angiography results
}

\author{
Asra Al Fauzi, Yunus Kuntawi Aji*, Nur Setiawan Suroto \\ Neurovascular Division, Department of Neurosurgery, Faculty of Medicine Universitas Airlangga/dr. Soetomo Academic Medical Center Hospital, Surabaya, Indonesia
}

\section{A R T I C L E I N F O}

Article history:

Received 21 February 2019

Accepted 16 July 2019

() 2019 Elsevier Ltd. All rights reserved.

\section{Introduction}

The middle cerebral artery (MCA) is the largest and most complex of cerebral arteries because the cerebral neocortex is significantly developed in humans [1,2]. The MCA covers a large part of the cerebral hemispheres; therefore, it is exposed during surgical intervention in that area. Aspects of the cerebral branches tend to vary, and different branching patterns can be described. In the past, surgical interest in the MCA has been directed at avoiding damage to its branches during surgery performed within its territory. Microsurgical techniques have made reconstruction and bypass to the MCA, surgical approaches to MCA aneurysms and resection of arteriovenous malformations (AVMs) related to MCA branches common procedures in vascular neurosurgery [2].

The vascular territory of the MCA includes some of the most eloquent cortical areas for motor and sensory functions. That territory encompasses the receptive and expressive components of language, abstract thought and other faculties of higher cognitive functioning. Moreover, the perforating branches of the proximal MCA supply the basal ganglia and important descending and corticospinal tracts [3]. Anatomical variations of the MCA have to be recognised when planning interventions in order to avoid damage or occlusion of the perforating vessels that arise from the MCA and to assess their contribution to the perfusion of the deep MCA territory [4].

Descriptions of the origins and possible common trunks of the MCA branches are still lacking in the literature. While bifurcation and trifurcation types of MCA branching are usually described, most studies fail to mention the different subtypes. Moreover, there is still some confusion about the criteria used to determine the different branching subtypes. Thus, the present study aimed to review the neuroangiography patterns of the MCA.

\footnotetext{
* Corresponding author.

E-mail address: yunus.neurosurgery@gmail.com (Y.K. Aji).
}

\section{Patients and methods}

\subsection{Patients and neuroangiography data}

Data were retrieved from the Neuroangiography Center of Dr Soetomo Academic Medical Center Hospital database, which includes all the neurosurgery patients who underwent cerebral angiography during the 2014-2018 period. We identified data from 578 consecutive cerebral angiographies. We excluded the results from 24 cases because of a lack of adequate MCA angiographies $(n=12)$, broken data $(n=4)$ and the lack of data on MCA patterns (posterior circulation data only) $(n=8)$. The remaining 554 cerebral angiography results from 300 patients had adequate MCA angiography patterns. The use of cerebral angiography in our hospital began in 2014; since then, it has been the imaging modality used for many of the neurosurgical cases at our institution. Each patient's neuroangiography images were stored in the database in the Digital Imaging and Communications in Medicine (DICOM) data format. The DICOM data were reviewed using DICOM reader software (Horos ${ }^{\mathrm{TM}}$ ) (measurement was done using the length calculation feature in the software) and analysed with IBM SPSS ${ }^{\circledR}$ software using descriptive statistics.

\subsection{Nomenclature}

The MCAs were identified in each patient and classified. The length of the main trunk was classified as short (3-12 mm), medium (13-22 mm) or long (23-40 mm). If branching occurs within $5 \mathrm{~mm}$ from the MCA origin, it is referred to as early branching [5].

The MCAs were grouped into 11 branching patterns: (1) monofurcation, (2) tetrafurcation, (3) pseudotetrafurcation, (4) medial bifurcation, (5) lateral bifurcation, (6) medial pesudobifurcation, (7) lateral pseudobifurcation, (8) true trifurcation, (9) pseudotrifurcation, (10) proximal trifurcation and (11) distal trifurcation (Fig. 1) [6]. 


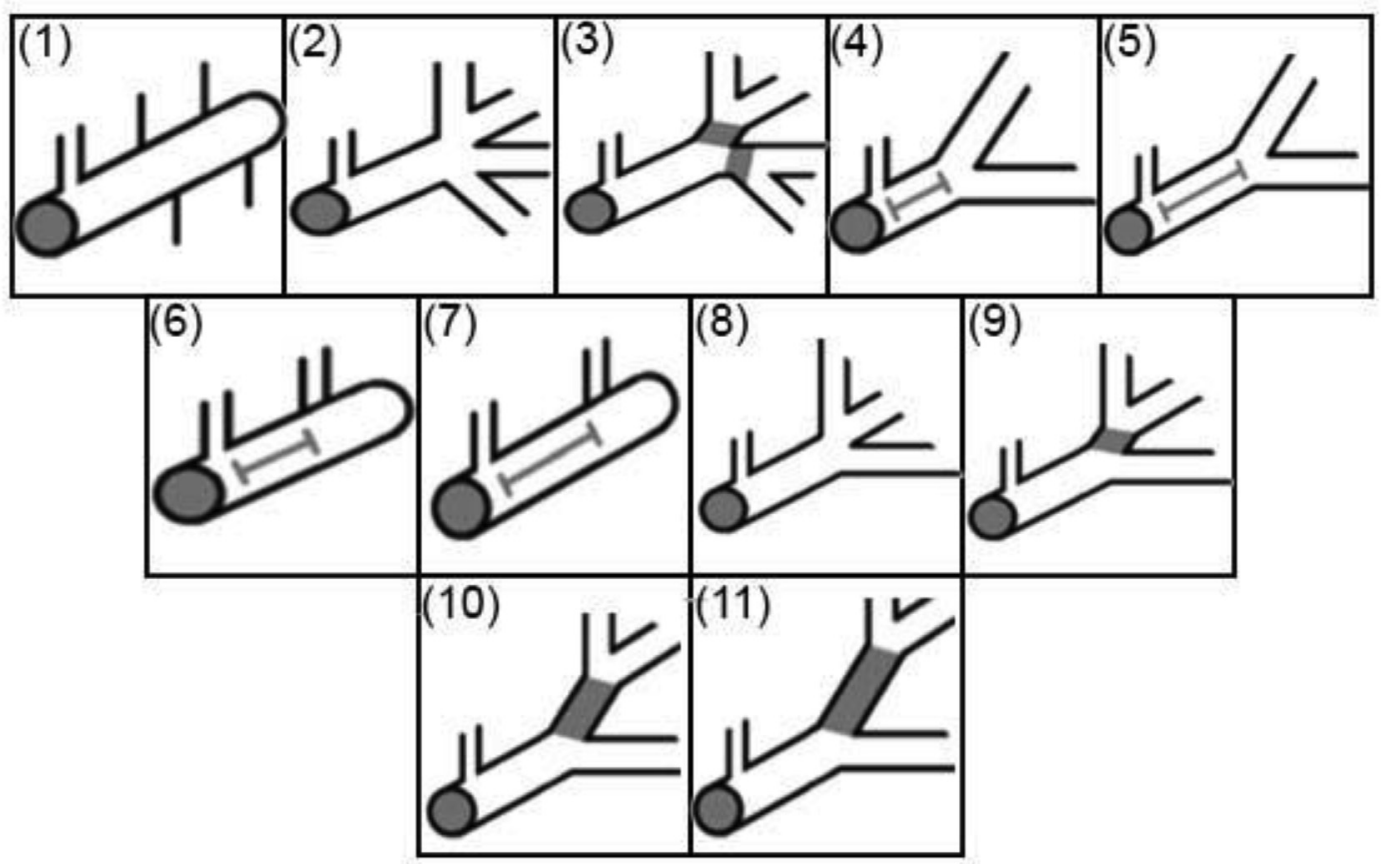

Fig. 1. The 11 different branching patterns of MCAs.

Table 1

Baseline variables of cerebral angiography results.

\begin{tabular}{lll}
\hline Variables & Total $(\mathrm{n}=300), \mathrm{n}(\%)$ & Remarks \\
\hline Age, y.o & & Mean \pm SD $=37.3 \pm 17.3$ \\
$<18$ & $48(16 \%)$ & \\
$\geq 18$ & $252(84 \%)$ & \\
Sex & & \\
Male & $125(41.7 \%)$ & \\
Female & $175(58.3 \%)$ & \\
Diagnosis & & \\
Aneurysm & $53(17.6 \%)$ & \\
AVM & $39(13 \%)$ & \\
Stenosis/infarct & $13(4.3 \%)$ & \\
CCF & $31(10.3 \%)$ & \\
Stroke ICH & $16(5.3 \%)$ & \\
Trauma & $8(2.7 \%)$ & \\
dAVF & $6(2 \%)$ & Parkinson with cerebellar \\
Moya-moya disease & $4(1.3 \%)$ & degeneration, Cerebellar \\
Thrombosis & $2(0.7 \%)$ & atrophy \\
Other & $2(0.7 \%)$ & \\
& & \\
Unknown & $34(11.3 \%)$ & \\
Side & $(\mathrm{n}=554)$ & \\
Right & $286(51.6 \%)$ & \\
Left & $268(48.4 \%)$ & \\
\hline & & \\
\hline & & \\
& &
\end{tabular}

\section{Results}

\subsection{Demographics}

From the baseline variables (Table 1 ), the mean age of the subjects was 37.3 years. Most of the subjects (252 subjects; $84 \%$ ) were adults ( $\geq 18$ years old). The male-to-female ratio was 5:7 (41.7\%:58.3\%). Aneurysm was the most frequent diagnosis (53 results, $17.6 \%$ ), followed by AVM (39 results, 13\%), carotidcavernous fistula (CCF) (31 results, 10.3\%), stroke intracerebral haemorrhage ( $\mathrm{ICH})$ (16 results, 5.3\%), stenosis/infarct (13 results, $4.3 \%$ ), traumatic brain injury (TBI) ( 8 results, $2.7 \%$ ), dural arteriovenous fistula (DAVF) (6 results, $2 \%$ ), Moyamoya disease (4 results,
Table 2

The MCA patterns from cerebral angiography results.

\begin{tabular}{lll}
\hline Variables & Total $(\mathrm{n}=554), \mathrm{n}(\%)$ & Remarks \\
\hline $\begin{array}{l}\text { Diameter, mm } \\
\text { Length, mm }\end{array}$ & $\begin{array}{l}\text { Mean } \pm \text { SD }=2.39 \pm 0.49 \\
\text { Lean } \pm \text { SD }=15.56 \pm 7.75\end{array}$ \\
Short $(3-12 \mathrm{~mm})$ & $225(40.6 \%)$ & \\
Medium $(13-22 \mathrm{~mm})$ & $220(39.7 \%)$ & \\
Long $(23-40 \mathrm{~mm})$ & $109(19.7 \%)$ & \\
Early Branching $(\leq 5 \mathrm{~mm})$ & & \\
Yes & $33(6 \%)$ & \\
No & $521(94 \%)$ & \\
Branching Pattern & & \\
Monofurcation & $0(0 \%)$ & \\
Tetrafurcation & $4(0.7 \%)$ & \\
Pseudotetrafurcation & $4(0.7 \%)$ & \\
Medial bifurcation & $255(46 \%)$ & \\
Lateral bifurcation & $67(12.1 \%)$ & \\
Medial pseudotrifurcation & $14(2.5 \%)$ & \\
Lateral pseudotrifurcation & $4(0.7 \%)$ & \\
True trifurcation & $52(9.4 \%)$ & \\
Pseudotrifurcation & $27(4.9 \%)$ & \\
Proximal trifurcation & $69(12.5 \%)$ & \\
Distal trifurcation & $58(10.5 \%)$ & \\
\hline
\end{tabular}

$1.3 \%$ ), sinus thrombosis (2 results, $0.7 \%$ ) and other (2 results, $0.7 \%$, including Parkinson's with cerebellar degeneration and cerebellar atrophy). Unfortunately, the diagnoses from 34 results (11.3\%) were not included in the database, so they were not available. According to the side of the MCAs, the right-to-left ratio is nearly equal (51.6\%: $48.4 \%)$.

\subsection{Patterns of MCAs}

Based on the MCA pattern results (Table 2), the mean diameter of the MCA (M1) was $2.39 \mathrm{~mm}$; the mean length was $15.56 \mathrm{~mm}$. Based on the classification for the length of the main trunk, most of the MCAs were short (225 results, 40.6\%) (Fig. 2). Medium MCAs (Fig. 3) and long MCAs (Fig. 4) were seen in 220 results (39.7\%) and 109 results (19.7\%), respectively. Early branching was only found in 


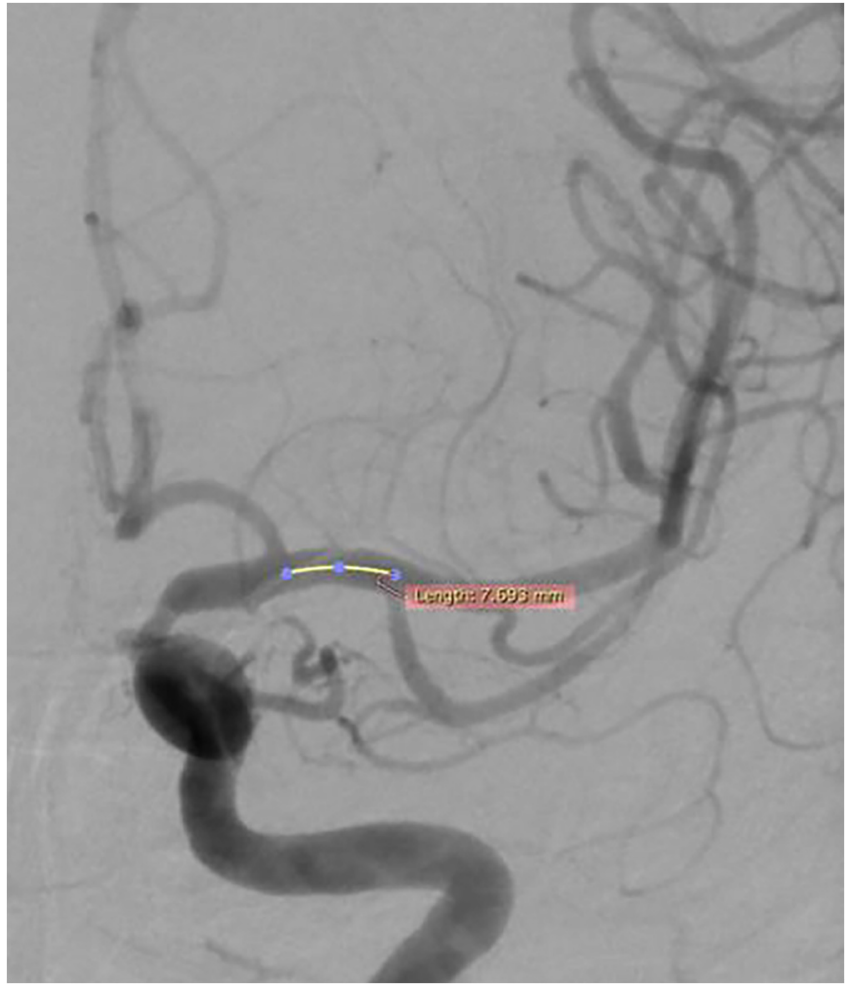

Fig. 2. Short segment.

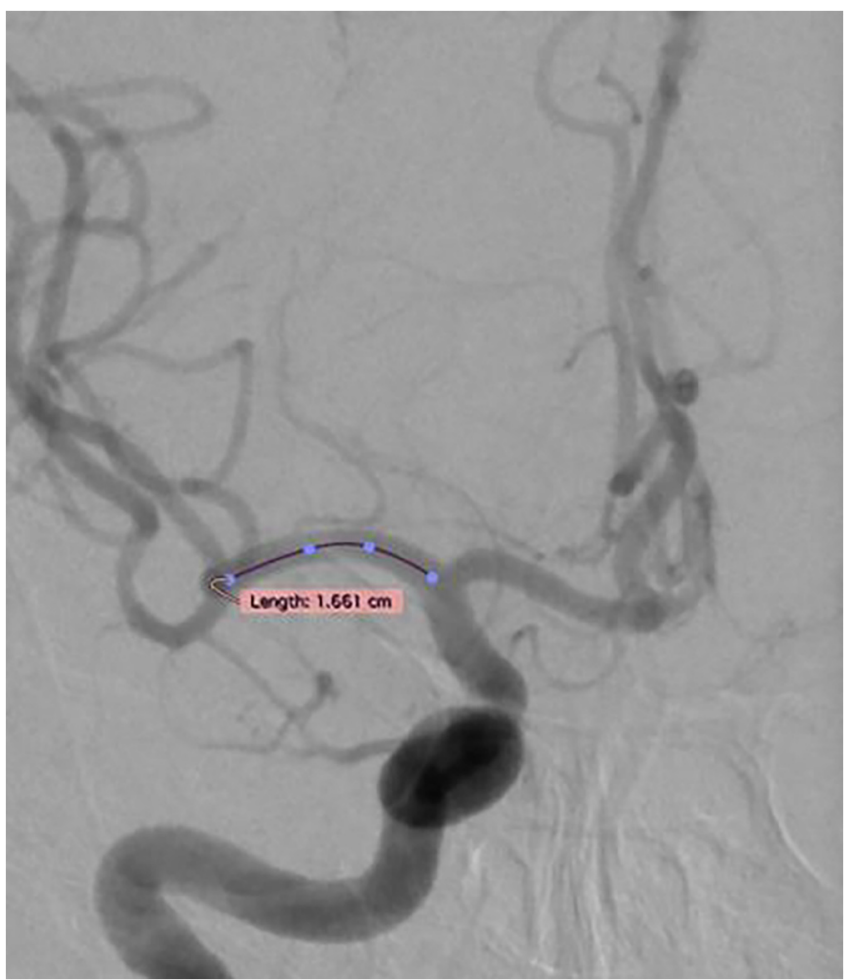

Fig. 3. Medium segment.

33 of the results (6\%) (Fig. 5). The most frequent branching pattern was medial bifurcation (255 results, 46\%) (Fig. 6), followed by proximal trifurcation (69 results, 12.5\%) (Fig. 7), lateral bifurcation (67 results, 12.1\%) (Fig. 8), distal trifurcation (58 results, 10.5\%) (Fig. 9), true trifurcation (52 results, 9.4\%) (Fig. 10), pseudotrifurca-

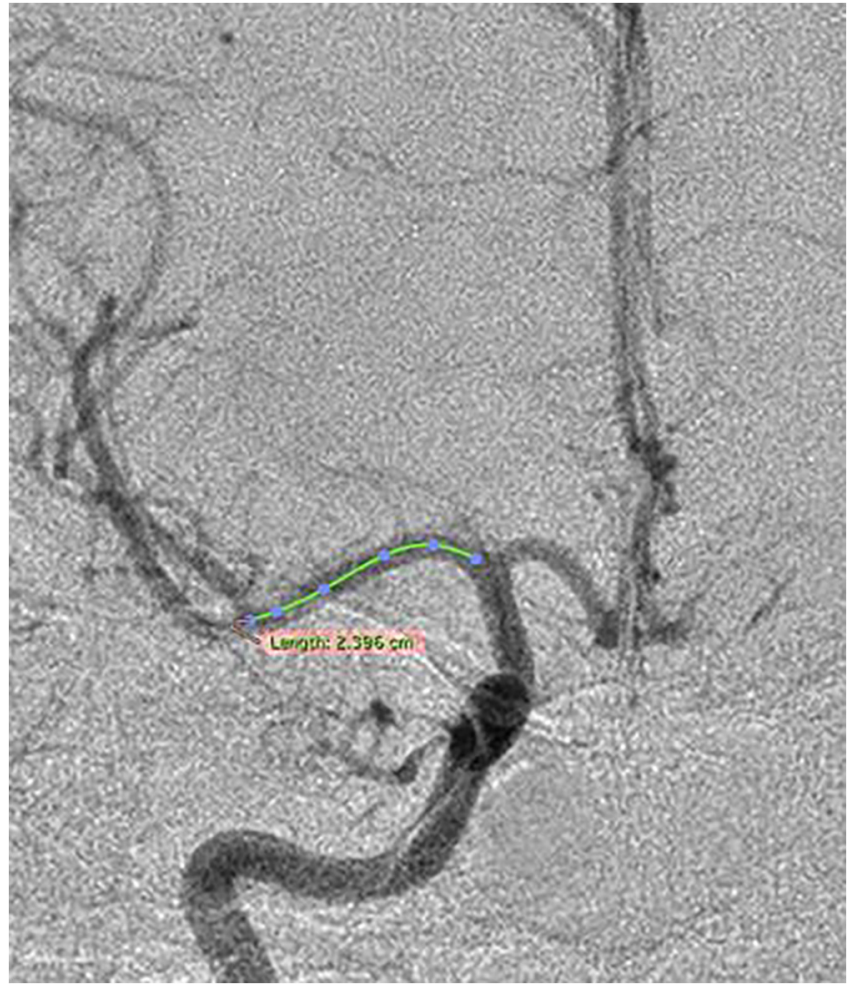

Fig. 4. Long segment.

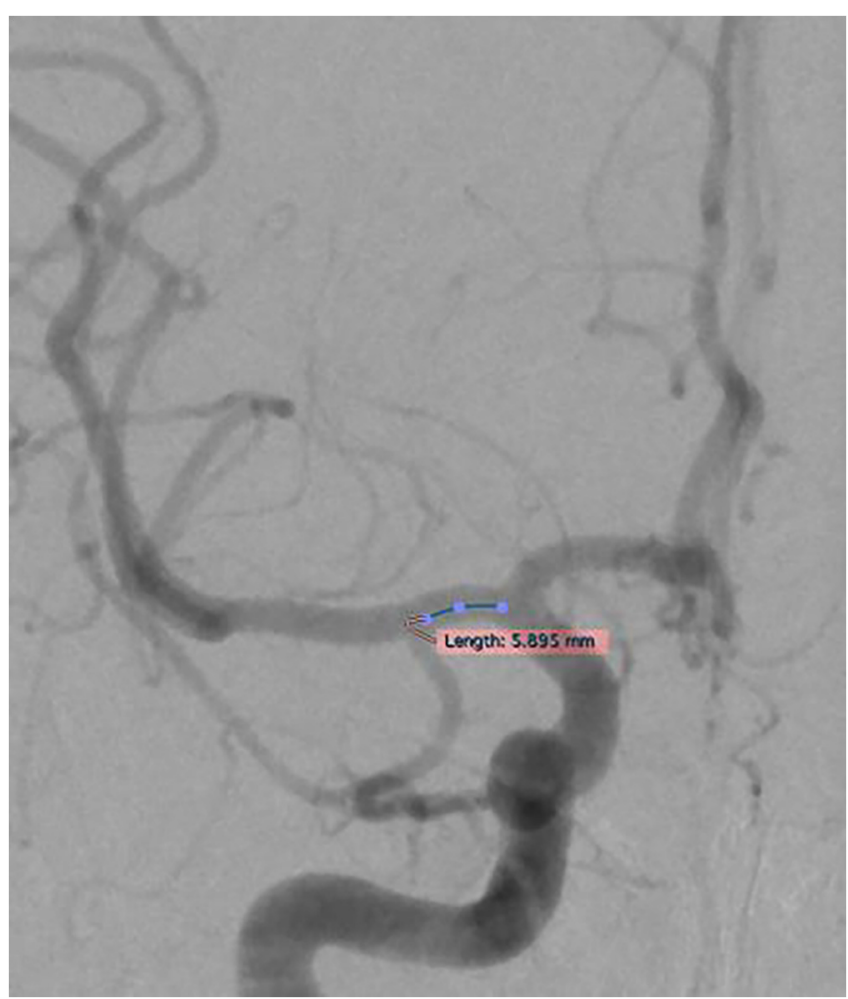

Fig. 5. Early branching.

tion (27 results, 4.9\%) (Fig. 11), medial pseudobifurcation (14 results, 2.5\%) (Fig. 12), lateral pseudobifurcation (4 results, 0.7\%) (Fig. 13), tetrafurcation (4 results, $0.7 \%$ ) (Fig. 14) and pseudotetrafurcation (4 results, $0.7 \%$ ) (Fig. 15). We did not find any monofurcation in this study. 


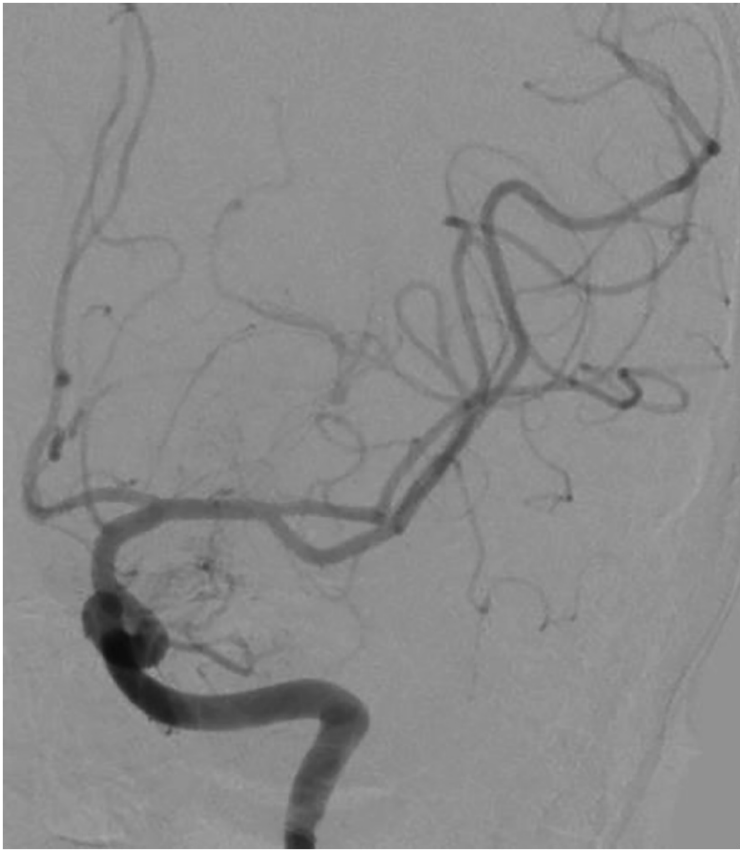

Fig. 6. Medial bifurcation.

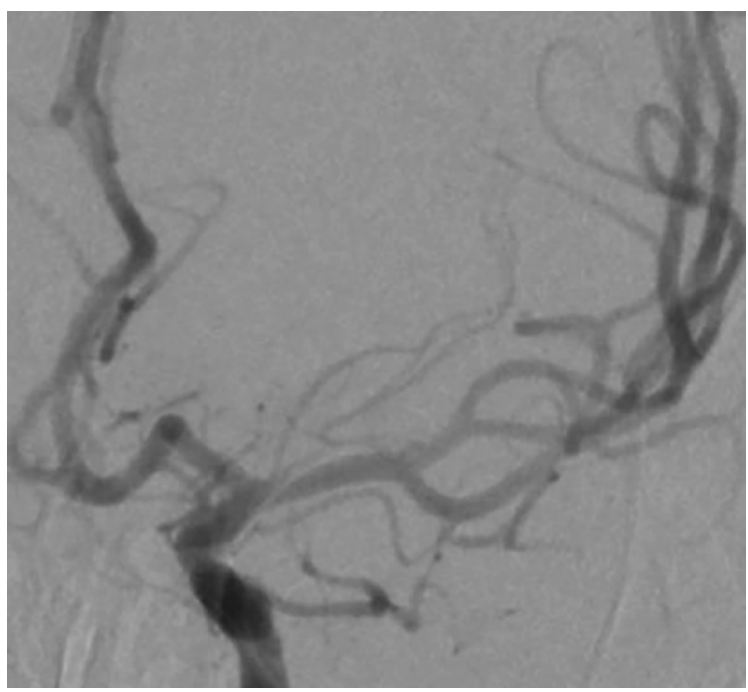

Fig. 7. Proximal trifurcation.

\subsection{Estimation in the population}

Based on the MCA pattern results (Table 2), we estimated the mean diameter and the mean length of the MCAs in the study population (Table 3). The estimation of the MCA (M1) mean diameter in the population was $2.39-2.43 \mathrm{~mm}$; the estimation of the mean length was 14.91-16.21 mm. A short MCA was estimated to occur in approximately $36.5-33.8 \%$ of the population, a medium MCA was estimated to occur in approximately $35.6 \%-43.9 \%$ of the population and a long MCA was estimated to occur in approximately $16.4 \%-23.2 \%$ of the population. The percentage early branching was estimated to be approximately $4.1 \%-8.3 \%$.

The estimation of the MCA branching patterns in the population were as follows: monofurcation $(0 \%-0.7 \%)$, tetrafurcation $(0.2 \%-$ $1.8 \%)$, pseudotetrafurcation $(0.2 \%-1.8 \%)$, medial bifurcation (41.8\%-50.3\%), lateral bifurcation (9.5\%-15.1\%), medial pseudotrifurcation $(1.4 \%-4.2 \%)$, lateral pseudotrifurcation $(0.2 \%-1.8 \%)$, true

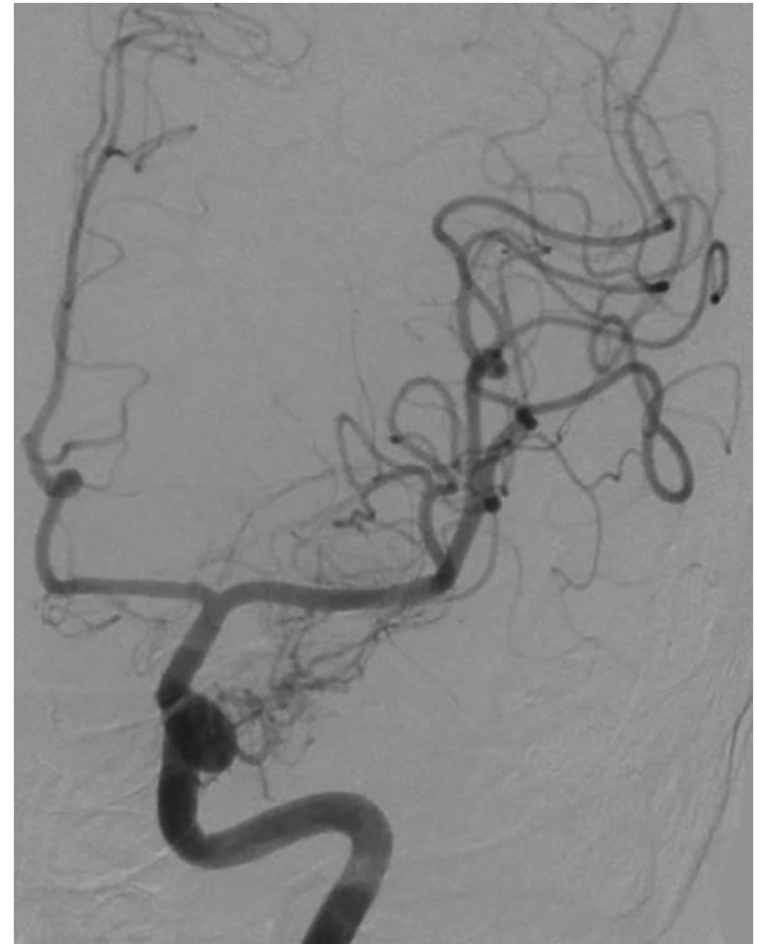

Fig. 8. Lateral bifurcation.

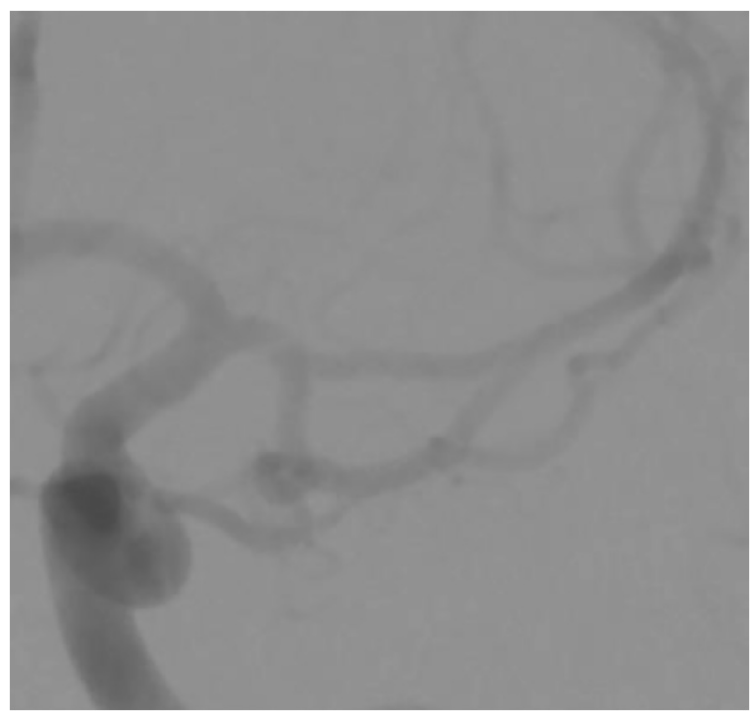

Fig. 9. Distal trifurcation.

trifurcation (7.1\%-12.1\%), pseudotrifurcation (3.2\%-7\%), proximal trifurcation (9.8\%-15.5\%) and distal trifurcation (8.0\%-13.3\%).

\section{Discussion}

In the present study, the mean diameter of the MCA (M1) was $2.39 \pm 0.49 \mathrm{~mm}$. This result was similar to the finding reported by Lasjaunias et al., which stated that the MCA diameter ranged from $2.4 \mathrm{~mm}$ to $4.6 \mathrm{~mm}$ [7]. In the present study, the length of the MCA (M1) was $15.56 \pm 7.75 \mathrm{~mm}$. This result is also similar to the findings reported by Harrigan et al., which stated that the average M1 segment is $16 \mathrm{~mm}$ long [8]. 


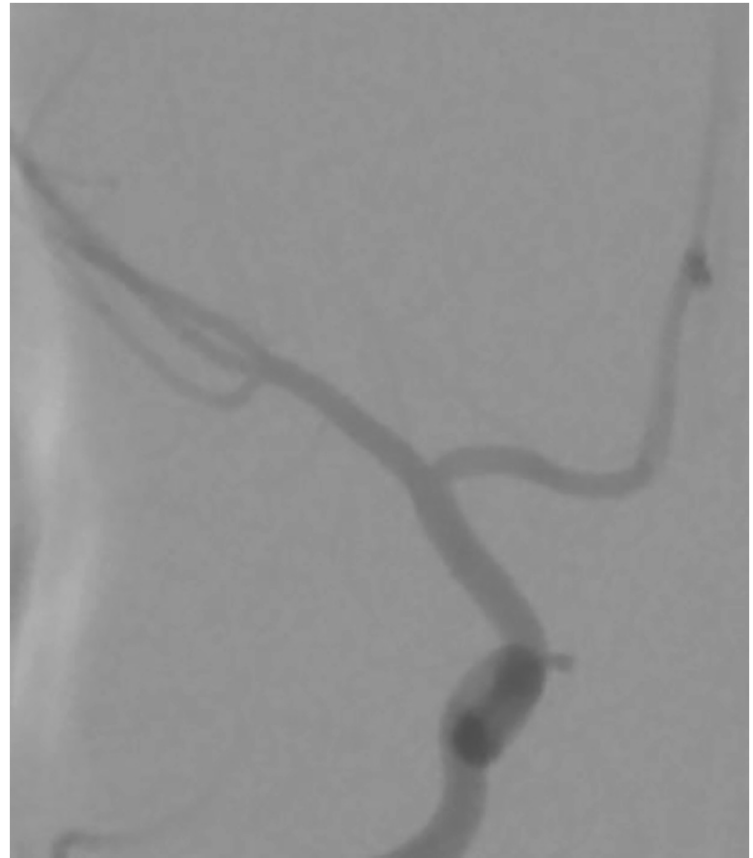

Fig. 10. True trifurcation.

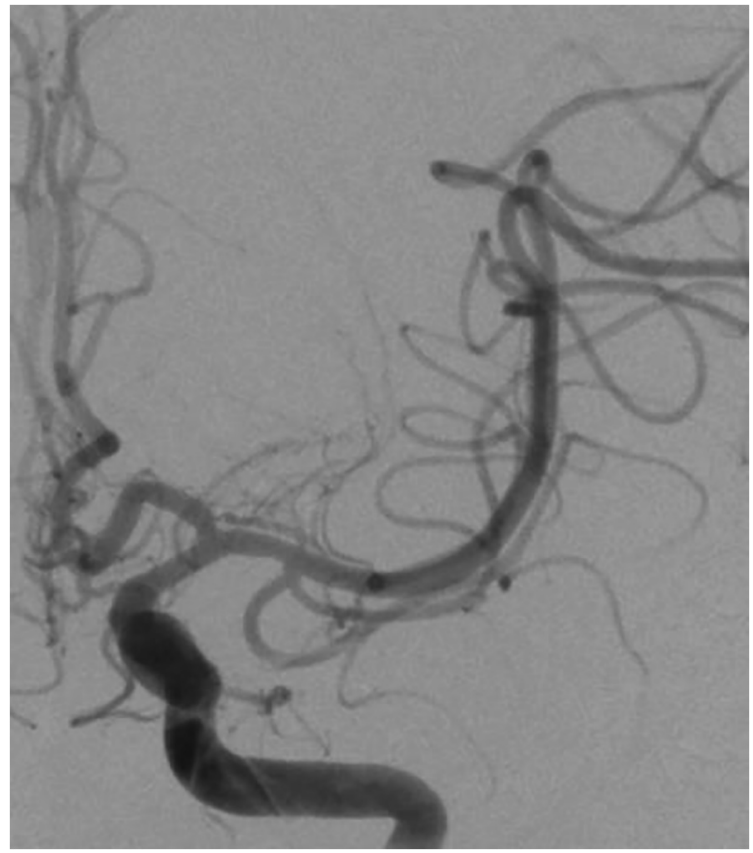

Fig. 11. Pseudotrifurcation.

Grellier et al. reported that the main trunk could be short (3$12 \mathrm{~mm}$ ), medium (13-22 mm) or long (23-40 mm) [6]. To the best of our knowledge, the percentages associated with this classification have never been published. In the present study, short MCAs, medium MCAs and long MCAs were seen in 40.6\%, 39.7\% and $19.7 \%$ of the cases, respectively.

Most authors agree that branching within $5 \mathrm{~mm}$ is referred to as early branching. In a previous in the literature, early branching was reported to range from $2.7 \%$ to $11.3 \%$; in the present study it was seen in $6 \%$ of the cases [6].

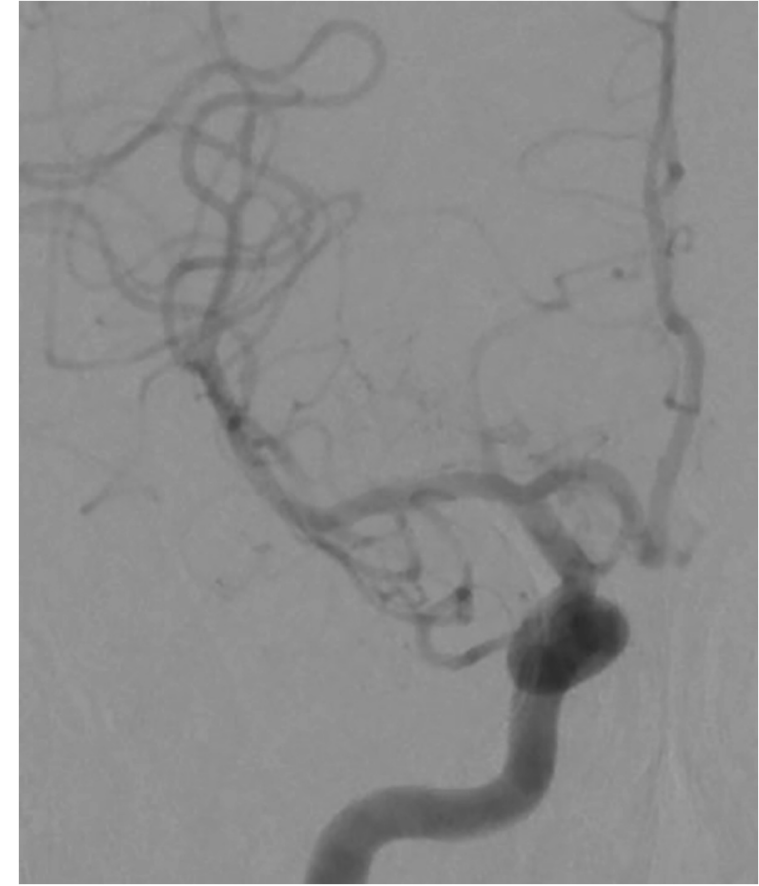

Fig. 12. Medial pseudobifurcation.

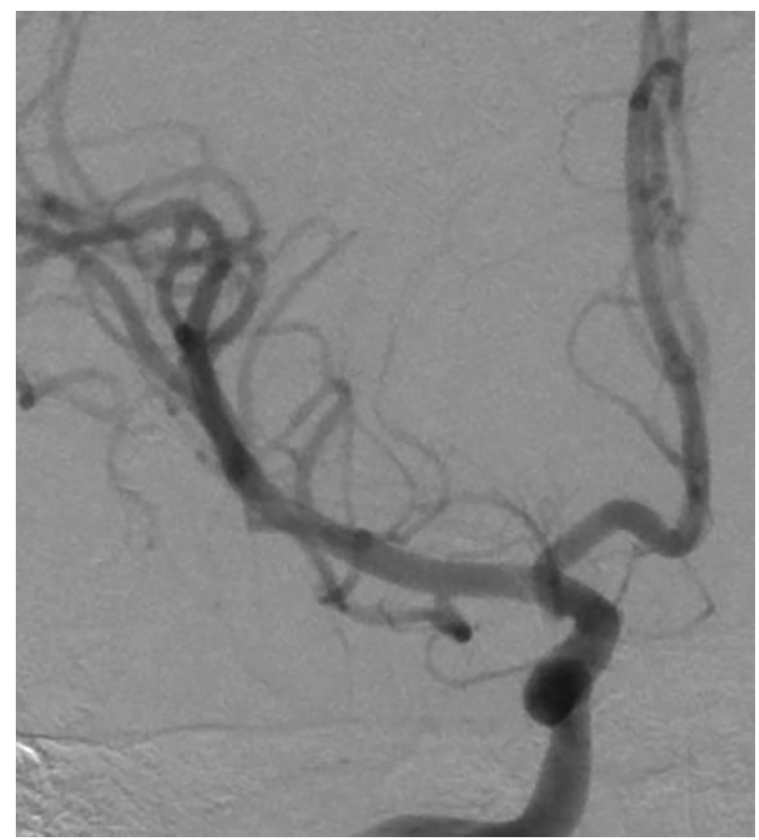

Fig. 13. Lateral pseudobifurcation.

In this study, we refer to 11 different MCA patterns as classified by Cilliers et al. [6]. In the present study, medial bifurcation (46\%) was the branching pattern that was most frequently found, followed by proximal trifurcation $(12.5 \%)$, lateral bifurcation (12.1\%), distal trifurcation (10.5\%), true trifurcation (9.4\%), pseudotrifurcation (4.9\%), medial pseudobifurcation (2.5\%), lateral pseudobifurcation $(0.7 \%)$, tetrafurcation $(0.7 \%)$ and pseudotetrafurcation $(0.7 \%)$. No monofurcation was found in this study. To the best of our knowledge, no previous study has reported on the percentages of MCA patterns based on these 11 different patterns. 


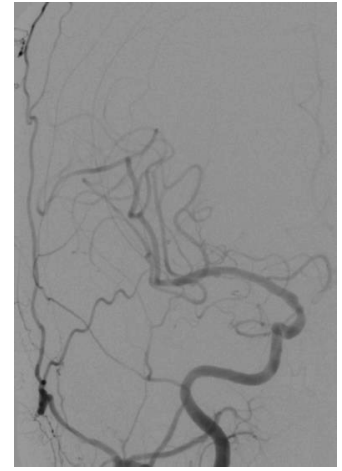

Fig. 14. Tetrafurcation.

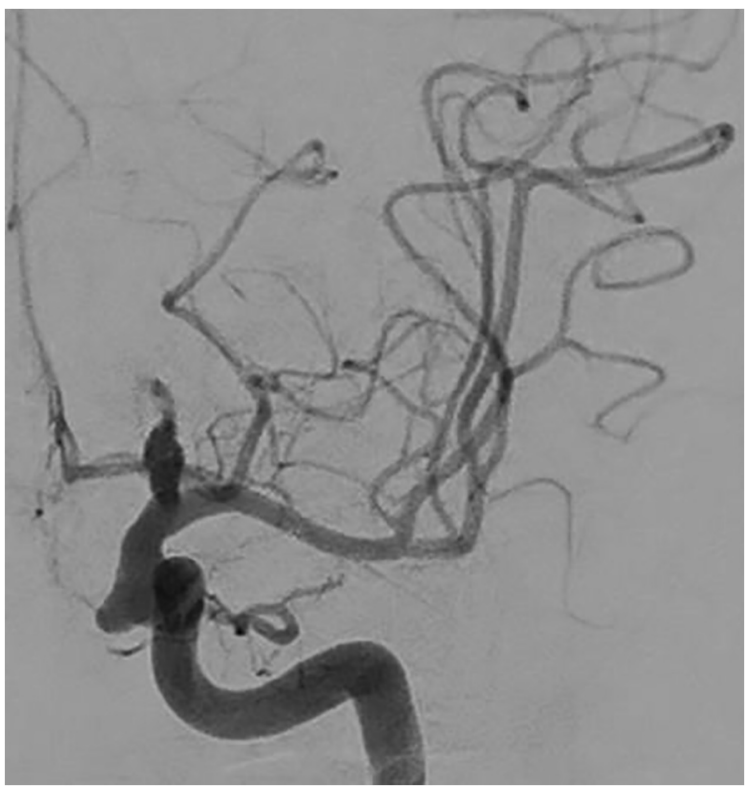

Fig. 15. Pseudotetrafurcation.

Table 3

Estimation of the MCA patterns in the population.

\begin{tabular}{lll}
\hline Variables & Total $(\mathrm{n}=554), \mathrm{n}(\%)$ & $95 \% \mathrm{CI}$ \\
\hline Diameter, mm & Mean $\pm \mathrm{SD}=2.39 \pm 0.49$ & $2.34-2.43$ \\
Length, mm & Mean $\pm \mathrm{SD}=15.56 \pm 7.75$ & $14.91-16.21$ \\
Length Type & & \\
Short $(3-12 \mathrm{~mm})$ & $225(40.6 \%)$ & $36.5 \%-44.8 \%$ \\
Medium $(13-22 \mathrm{~mm})$ & $220(39.7 \%)$ & $35.6 \%-43.9 \%$ \\
Long $(23-40 \mathrm{~mm})$ & $109(19.7 \%)$ & $16.4 \%-23.2 \%$ \\
Early Branching $(\leq 5 \mathrm{~mm})$ & & \\
Yes & $33(6 \%)$ & $4.1 \%-8.3 \%$ \\
No & $521(94 \%)$ & $91.7 \%-95.9 \%$ \\
Branching Pattern & & \\
Monofurcation & $0(0 \%)$ & $0 \%-0.7 \%$ \\
Tetrafurcation & $4(0.7 \%)$ & $0.2 \%-1.8 \%$ \\
Pseudotetrafurcation & $4(0.7 \%)$ & $0.2 \%-1.8 \%$ \\
Medial bifurcation & $255(46 \%)$ & $41.8 \%-50.3 \%$ \\
Lateral bifurcation & $67(12.1 \%)$ & $9.5 \%-15.1 \%$ \\
Medial pseudotrifurcation & $14(2.5 \%)$ & $1.4 \%-4.2 \%$ \\
Lateral pseudotrifurcation & $4(0.7 \%)$ & $0.2 \%-1.8 \%$ \\
True trifurcation & $52(9.4 \%)$ & $7.1 \%-12.1 \%$ \\
Pseudotrifurcation & $27(4.9 \%)$ & $3.2 \%-7 \%$ \\
Proximal trifurcation & $69(12.5 \%)$ & $9.8 \%-15.5 \%$ \\
Distal trifurcation & $58(10.5 \%)$ & $8.0 \%-13.3 \%$ \\
\hline
\end{tabular}

Table 4

The percentage of monofurcation, bifurcation, trifurcation, and tetrafurcation, with its average [7].

\begin{tabular}{lllll}
\hline Authors & $\begin{array}{l}\text { Monofur- } \\
\text { cation }\end{array}$ & Bifurcation & $\begin{array}{l}\text { Trifur- } \\
\text { cation }\end{array}$ & $\begin{array}{l}\text { Tetra- } \\
\text { furcation }\end{array}$ \\
\hline Jain (1964) [8] & $0 \%$ & $90 \%$ & $10 \%$ & $0 \%$ \\
Grellier (1978) [9] & $17.5 \%$ & $71.1 \%$ & $11.4 \%$ & $0 \%$ \\
Gibo (1981) [10] & $0 \%$ & $78 \%$ & $12 \%$ & $10 \%$ \\
Umansky (1984) [11] & $5.7 \%$ & $64.3 \%$ & $28.6 \%$ & $1.4 \%$ \\
Antunes (1985) [12] & $0 \%$ & $89.2 \%$ & $10.8 \%$ & $0 \%$ \\
Umansky (1985) [13] & $0 \%$ & $70.6 \%$ & $20.6 \%$ & $8.8 \%$ \\
Umansky (1988) [14] & $3.8 \%$ & $66.3 \%$ & $26 \%$ & $3.8 \%$ \\
Anderhuber (1990) [15] & $0 \%$ & $0 \%$ & $7 \%$ & $0 \%$ \\
Meneses (1997) [16] & $7.1 \%$ & $85.7 \%$ & $7.1 \%$ & $0 \%$ \\
Idowu (2002) [17] & $6 \%$ & $81 \%$ & $13 \%$ & $0 \%$ \\
Kulenovic (2003) [18] & $0 \%$ & $70 \%$ & $30 \%$ & $0 \%$ \\
Tanriover (2003) [19] & $0 \%$ & $88 \%$ & $12 \%$ & $0 \%$ \\
Tanriover (2004) [20] & $0 \%$ & $88.4 \%$ & $11.6 \%$ & $0 \%$ \\
Pai (2005) [21] & $0 \%$ & $80 \%$ & $0 \%$ & $0 \%$ \\
Vuillier (2008) [22] & $17 \%$ & $73 \%$ & $9 \%$ & $0 \%$ \\
Nowinski (2009) [23] & $0 \%$ & $78 \%$ & $12 \%$ & $0 \%$ \\
Ogeng'o (2011 [24] & $6.3 \%$ & $82.3 \%$ & $10.8 \%$ & $0.7 \%$ \\
Sadatomo (2013) [25] & $0 \%$ & $92.7 \%$ & $7.3 \%$ & $0 \%$ \\
Average & $3.5 \%$ & $74.9 \%$ & $13.2 \%$ & $1.3 \%$ \\
\hline
\end{tabular}

If we re-classified these patterns into monofurcation, bifurcation, trifurcation and tetrafurcation, the percentages for these patterns would be $0 \%, 61.3 \%, 37.3 \%$ and $1.4 \%$, respectively. As seen in Table 2 , these results can be compared to the results reported in previous studies [6]. Monofurcation was seen, on average, in 3.5\% cases (ranging from $0 \%$ to $17.5 \%$ ), bifurcation was seen, on average, in $74.9 \%$ cases (ranging from $0 \%$ to $92.7 \%$ ), trifurcation was seen, on average, in $13.2 \%$ cases (ranging from $0 \%$ to $30 \%$ ) and tetrafurcation was seen, on average, in $1.3 \%$ cases (ranging from $0 \%$ to $8.8 \%$ ) (Table 4).

\section{Conclusion}

Anatomical variations of the MCA have to be recognised when planning surgical and endovascular interventions. Studying the neuroangiography patterns of MCAs may help to plan an optimal treatment strategy.

\section{Disclosure of interest}

The authors declare that they have no competing interest.

\section{Funding}

This study was supported by the Institute of Research and Innovation (LPI) Universitas Airlangga (Penelitian Dosen Pemula grant).

\section{Appendix A. Supplementary data}

Supplementary data to this article can be found online at https://doi.org/10.1016/j.jocn.2019.07.054.

\section{References}

[1] Uchiyama N. Anomalies of the middle cerebral artery. Neurol Med-Chir Adv 2017:1-6.

[2] Almeida JPC, Chaddad F, Rhoton AL, Oliveira E. Cranial Vascular Anatomy of the Anterior Circulation. In: Spetzler RF, Kalani MYS, Nakaji P, editors. Neurovascular Surgery. New York: Thieme; 2015. p. 41-8. 75-9.

[3] Morris P. The Middle Cerebral Artery. In: In Practical Neuroangiography. Philadelphia: Lippincott Williams \& Wilkins; 2015. p. 100-7. 114-30, 175-81. 
[4] Krings T, Geibprasert S, Cruz JP, Terbrugge KG. The Middle Cerebral Artery Trunk. In: In Neurovascular Anatomy in Interventional Neuroradiology. New York: Thieme; 2015. p. 62-82.

[5] Grellier P, Roche JL, Duplay J. Radio-anatomical study of the main trunk of the middle cerebral artery. Neurochirurgie 1978;24(4):227-33.

[6] Cilliers K, Page BJ. Anatomy of the middle cerebral artery: cortical branches, branching pattern and anomalies. Turk Neurosurg 2016:1-11.

[7] Lasjaunias P, Berenstein A, Brugge KGT. Intradural Arteries. In: In Surgical Neuroangiography. New York: Springer; 2001. p. 613-29.

[8] Harrigan MR, Deveikis JP. Essential Neurovascular Anatomy. In: In Handbook of Cerebrovascular Disease and Neurointerventional Technique. New York: Springer; 2013. p. 54-8.

[9] Jain KK. Some observations on the anatomy of the middle cerebral artery. Can J Surg 1964;7:134-9.

[10] Gibo H, Carver CC, Rhoton AL, Lenkey C, Mitchell RJ. Microsurgical anatomy of the middle cerebral artery. J Neurosurg 1981;54:151-69.

[11] Umansky F, Juarez SM, Dujovny M, Ausman JI, Diaz FG, Gomes F, et al. Microsurgical anatomy of the proximal segments of the middle cerebral artery. J Neurosurg 1984;61:458-67.

[12] Antunes ACM. The microsurgical anatomy of the human middle cerebral artery. Arq Bras Neurochir 1985;4:195-208.

[13] Umansky F, Gomes FB, Dujovny M, Diaz FG, Ausman JI, Haresh G, et al. The perforating branches of the middle cerebral artery: a microanatomical study. J Neurosurg 1985;62:261-8.

[14] Umansky F, Dujovny M, Ausman JI, Diaz FG, Mirchandani HG. Anomalies and variations of the middle cerebral artery: a microanatomical study. Neurosurgery 1988;22(6 Pt 1):1023-7.

[15] Anderhuber F, Weiglein A, Pucher RK. Trifurcations of the middle cerebral arteries. Acta Anat 1990;137(4):342-9.
[16] Meneses MS, Ramina R, Jackowski AP, Pedrozo AA, Pacheco RB. Tsubouchi MH Middle cerebral artery revascularization. Anatomical studies and considerations on the anastomosis site. Arq Neuropsiquiatr 1997;55(1):16-23.

[17] Idowu OE, Shokunbi MT, Malomo AO, Ogunbiyi JO. Size, course, distribution and anomalies of the middle cerebral artery in adult Nigerians. East Afr Med J $2002 ; 79(4): 217-20$

[18] Kulenović A, Dilberović F. Ovcina F. Variation in the flow and branching of the anterior and middle cerebral arteries. Med Arch 2003;57(1):3-5.

[19] Tanriover N, Kawashima M, Rhoton AL, Ulm AJ, Mericle RA. Microsurgica anatomy of the early branches of the middle cerebral artery: Morphometric analysis and classification with angiographic correlation. J Neurosurg 2003:98:1277-90.

[20] Tanriover N, Rhoton AL, Kawashima M, Ulm AJ, Yasuda A. Microsurgical anatomy of the insula and the sylvian fissure. J Neurosurg 2004;100 (5):891922.

[21] Pai SB, Varma RG, Kulkarni RN. Microsurgical anatomy of the middle cerebral artery. Neurol India 2005;53(2):186-90.

[22] Vuillier F, Medeiros E, Moulin T, Cattin F, Bonneville JF, Tatu L. Main anatomical features of the M1 segment of the middle cerebral artery: A 3D time-of-flight magnetic resonance angiography at 3T study. Surg Radiol Anat 2008;30(6):509-14

[23] Nowinski WL, Thirunavuukarasuu A, Volkau I, Marchenko Y, Aminah B, Puspitasari $\mathrm{F}$, et al. A three-dimensional interactive atlas of cerebral arterial variants. Neuroinformatics 2009;7(4):255-64.

[24] Ogeng'o JA, Njongo W, Hemed E, Obimbo MM, Gimongo J. Branching pattern of middle cerebral artery in an African population. Clin Anat 2011:24(6):692-8.

[25] Sadatomo T, Yuki K, Migita K, Imada Y, Kuwabara M, Kurisu K. Differences between middle cerebral artery bifurcations with normal anatomy and those with aneurysms. Neurosurg Rev 2013;36(3):437-45. 